\title{
Determination of CIE chromaticity coordinates of colored lights produced by a projection color mixer*
}

\author{
PETER K. KAISER \\ York University, Downsview, Ontario, Canada
}

\begin{abstract}
A procedure for measuring the CIE chromaticity coordinates which avoids some of the problems encountered with the method reported by Murch (1972) is described.
\end{abstract}

In 1964, Riggs described a projection color mixer designed for laboratory and classroom demonstrations of color-vision phenomena; Murch and Hirsch (1972) used a projection color mixer, similar in design, to match the chromatic-contingent aftereffects known as the "McCollough effect." (The apparatus is described in Murch, 1972.) More recently, Riggs, White, and Eimas (1973) used a color-mixing project or with a null method to measure orientation-contingent aftereffects of color, but did not describe the color-mixing device. Since Riggs originally used the projection color mixer for demonstrational purposes (1964), it may be useful to reexamine the device as an apparatus for collecting scientific color-vision data.

If the Riggs color mixer is used to quantify the perception of color by representing matches on the CIE chromaticity diagram, a means for computing the $\mathrm{x}, \mathrm{y}$ coordinates is required. Murch (1972) described a procedure, taken from Wyszecki and Stiles (1967), which requires knowledge of the following: (1) color temperature of projection lamp; (2) spectral transmittance of heat filter; (3) spectral transmittance of the three primary color filters; (4) spectral sensitivity of photosensor used to measure the light.

Murch explained the method for incorporating these factors into the appropriate colorimetric formulae to determine the CIE $x, y$ coordinates, but three additional factors were not considered: spectral transmittance of coated lenses, spectral reflectance of projection screen, and interreflection between three-part filters and the projection lens.

I recently constructed a projection color mixer using the instructions provided in the Riggs (1964) and Murch (1972) papers, and I found them to be deficient in one important respect: When forming an image of the source on the three filters, considerable stray light fell outside of the area covered by the filament image. Using the spectroradiometric measurements described below, it became evident that this stray light was important. Therefore, I placed an aperture, the size of a filament

\footnotetext{
*Supported by Grant A0295 from the National Research Council of Canada. I would like to thank G. Wyszecki for his comments.
}

image, between the projection lens and the mechanical stage holding the three filters.

This stray light would be crucial in the procedures described by Murch. He measured "the amount of light passing through each filter in the mounting stage after a specific color has been produced." Specifically, he measured the light going into the filters and the amount coming out of each filter. Presumably, his photodetector is designed to capture the light associated with that coming from the filament image alone and thus not necessarily any light coming from any part of the filters not directly associated with the area of the filament image.

A more direct method for determining the CIE x,y chromaticity coordinates measures the relative spectral radiant power of the color stimuli produced by the projection color mixer ${ }^{1}$. With this method, the entire system (color mixer and projection screen) is evaluated. Once the relative spectral radiant power of the color is known, the CIE-tristimulus values are computed according to Eq $1 \mathrm{a}-\mathrm{c}$ and $2 \mathrm{a}-\mathrm{b}$, described in Wyszecki and Stiles (1967).

$$
\begin{gathered}
\mathrm{X}=\mathrm{k} \sum_{\lambda} \mathrm{S}(\lambda) \overline{\mathrm{x}}(\lambda) \Delta \lambda \\
\mathrm{Y}=\mathrm{k} \sum_{\lambda} \mathrm{S}(\lambda) \overline{\mathrm{y}}(\lambda) \Delta \lambda \\
\mathrm{Z}=\mathrm{k} \sum_{\lambda} \mathrm{S}(\lambda) \overline{\mathrm{z}}(\lambda) \Delta \lambda
\end{gathered}
$$

$\mathrm{k}$ is a normalizing factor, $S(\lambda)$ is the relative radiant power, and $\bar{x}(\lambda), \bar{y}(\lambda), \bar{z}(\lambda)$ are the appropriately chosen color-matching functions. There are three choices of color-matching functions: (1) 1931 CIE color-matching scheme; (2) Judd's modification of the CIE color-matching functions ${ }^{2}$ (these two sets of functions are appropriate for field sizes of up to $4 \mathrm{deg}$ visual angle); and (3) for field sizes larger than $4 \mathrm{deg}$, the 1964 supplementary color-matching functions are appropriate.

Once the tristimulus values are known, they are converted to chromaticity coordinates according to the following formulae: 
Table 1

Measured and Kodak's Specification of CIE Chromaticity Coordinates of Wratten Filters and the Measured Chromaticity Coordinates of a Neutral White Light Reflected from Two Types of Projection Screens

\begin{tabular}{lcccc}
\hline & \multicolumn{2}{c}{ Measured } & \multicolumn{2}{c}{ Kodak B-3 } \\
& $\mathrm{x}$ & $\mathrm{y}$ & $\mathrm{x}_{\mathrm{A}}$ & $\mathrm{y}_{\mathrm{A}}$ \\
\hline Wratten 61 & .260 & .693 & .246 & .699 \\
Wratten 29 & .713 & .287 & .712 & .288 \\
Wratten 47 & .134 & .076 & .137 & .072 \\
Neutral White, White Screen & .355 & .371 & - & - \\
Neutral White, Aluminized Screen & .336 & .358 & - & - \\
\hline
\end{tabular}

$$
\begin{aligned}
& x=\frac{X}{X+Y+Z} \\
& y=\frac{Y}{X+Y+Z}
\end{aligned}
$$

Wyszecki and Stiles (1967, pp.322-323) provide an example of these computations.

The advantages of working from the reflected spectral radiant power are that no assumptions are required except those concerning the spectral sensitivity of the spectroradiometer and that a constant-current drives the projection lamp to maintain a constant-color temperature. Kodak Publication B-3 gives specifications for the stability of the various Wratten filters. The Wratten 61 (green) and 48 (blue) used by Murch are noted as somewhat unstable to tungsten light. The tungsten filament is imaged on the filters in the Murch color mixer. With the calibration method proposed in the present paper, one needs only to assume that the spectral transmittance of the. filters at the time of calibration are the same as when the color mixer was used. No assumption is required regarding the published specifications. Further, no measurements or assumptions of the spectral transmittances of the lenses and heat filters or spectral reflectances of the projection screen need to be made. Finally, when one calibrates the radiant energy reflected from the projection screen, no measurement or assumption regarding the efficacy of the interreflections between the Wratten filters and projection optics and within the projection optics need to be made.

Using the procedure discussed above, I determined the chromaticity coordinates of the three filters used in my projection color mixer. ${ }^{3}$ Table 1 shows the results and the Kodak specifications of the $\mathrm{x}, \mathrm{y}$ coordinates assuming CIE Source A.

These determinations were made by projecting the light through each filter individually onto a white nonbeaded projection screen. The obtained values were reasonably close to those published in Kodak Publication B-3. The color mixer was also set to yield a neutral white light when projected onto a white nonbeaded projection screen (i.e., a white light in which no hue could be detected by two Os). ${ }^{4}$ Spectroradiometry was then performed on the light reflected from a white nonbeaded screen, and an aluminized projection screen, respectively. The associated correlated color temperature from the white screen was $4,650^{\circ} \mathrm{K}$, from the aluminized screen it was $5,405^{\circ} \mathrm{K}$. These correlated color temperatures were determined by locating the mired value associated with the measured chromaticity coordinates of each white light from Fig. 1.30 in Wyszecki and Stiles (1967), converting these mired values by means of Table 1.18 in Wyszecki and Stiles to the correlated color temperature. The white screen seems to absorb more of the short wavelength radiation than does the aluminized screen. This difference in correlated color temperature of approximately $750^{\circ} \mathrm{K}$ demonstrates that the nature of the reflecting surface can be an important factor.

The procedures outlined both in this paper and in Murch (1972) described the manner in which a color stimulus can be plotted in the CIE chromaticity diagram. It sometimes is of interest to specify the dominant wavelength and excitation purity of a colored sample. Excitation purity is a ratio of the distance between a white point and the colored sample to the distance between the white point and the spectrum locus. If a desaturated light is obtained by mixing a white light with a monochromatic light lying on the spectrum locus, the computation is quite simple and is expressed according to Eq. 3 :

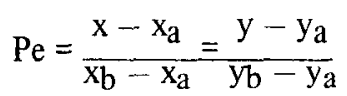

where $\mathrm{x}$ and $\mathrm{y}$ are the chromaticity coordinates of a given sample, $x_{a}$ and $y_{a}$ the coordinates of the white light, and $x_{b}$ and $y_{b}$ the coordinates of the monochromatic light on the spectrum locus. It is possible to calculate (Eq. 3) the excitation purity of a sample of unknown spectral composition, but of known position in the CIE diagram, by making an arbitrary assumption of a white light. A convenient assumption would be an equal-energy white $(\mathrm{x}=.33, \mathrm{y}=.33)$. The dominant wavelength is located by drawing a straight line from the equal-energy white point through the sample to the spectrum locus. The intersection of this line and the spectrum locus defines the dominant wavelength. (Fig. 1.)

If a given colored light is obtained with a projection color mixer, it will plot some place in the chromaticity space not on the spectrum locus. The way in which the dominant wavelength and excitation purity is computed for this colored light is to define a reference white point from which to make the calculations noted above. If the projection color mixer is used to obtain a colored light 


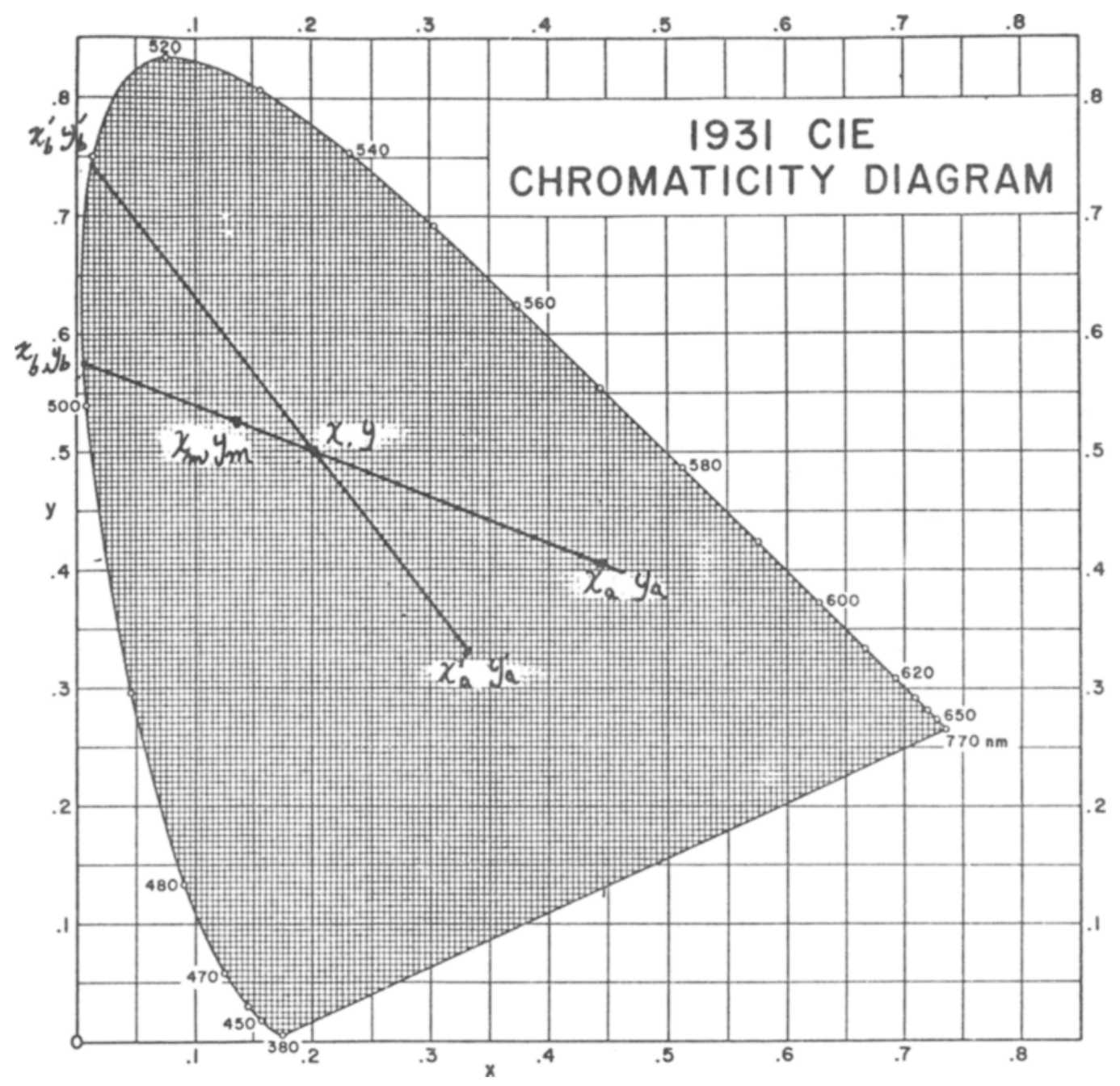

Fig. 1. 1931 CIE chromaticity diagram.

which is then desaturated by mixing it with a known white light, the chromaticity coordinates of this known white light can be used as the reference white point in the chromaticity space in order to compute dominant wavelength and excitation purity. But there is nothing absolute in choosing the chromaticity coordinates of this additional white light as the reference white point, because the colored light from the color mixer does not lie on the spectrum locus and thus is already desaturated to some extent. So, some white light must be used as a reference white point. Since we start with a sample not located on the spectrum locus, the addition of a known white light to bring the colored sample still further away from the locus becomes an arbitrary, but logical, choice with which to calculate the dominant wavelength and excitation purity.

My point concerning an arbitrary white light can be made in another way. Assume that we have a sample, $x, y$ in Fig. 1. This sample is matched with a white light $\left(\mathrm{x}_{a}^{\prime}\right.$, $y_{a}^{\prime}$ ) plus a monochromatic light ( $x_{b}^{\prime}, y_{b}^{\prime}$ ), which automatically is the dominant wavelength. Equation 3 is used to compute the excitation purity. If sample $x, y$ is matched with the color mixer, $x_{m}, y_{m}$ plus white light $x_{a}, y_{a}$. The dominant wavelength can be determined by extending a line from $x_{a}, y_{a}$ through $x_{m}, y_{m}$ to the spectrum locus $x \mathrm{~b}, \mathrm{y}_{\mathrm{b}}$. The excitation purity is determined by Eq. 3. We now have a sample $x, y$ matched by two fields of different spectral distribution. The sample and two matching fields are called metamers. Indeed, not only is the specification of the white arbitrary, but so are the specifications of the color-mixer primaries. If two investigators use the same white light but different filters in their projection color mixer, the resulting dominant wavelengths and excitation purities will be different for metameric matches.

This is not really a problem. The CIE diagram is intended only as a means for plotting and describing physical stimuli. The relation between values of stimuli 
plotted in the chromaticity space usually only bear some ordinal relationship to visual perception. The clearest exception is that the colors lying on the spectrum locus all have an excitation purity of 1.0 . However, the saturations of the colors lying on the spectrum locus are not constant.

\section{REFERENCES}

Murch, G. M. CIE $x, y$ coordinates from an inexpensive projection colorimeter. Behavior Research Methods \& Instrum entation, $1972,4,3-5$

Murch, G. M., \& Hirsch, J. The McCollough effect created by complementary afterimages. American Journal of Psychology, $1972,85,241-247$.

Riggs, L. A. A projection color mixer. American Journal of Psychology, 1964, 77, 129-134.

Riggs, L. A., White, K. D., \& Eimas, P. D. Encoding and decay of the orientation-contingent aftereffects of color. Journal of the Optical Society of America, 1973, 63, 1287. (Ab stract)
Wyszecki, G. Matching color differences. Journal of the Optical Society of America, 1965, 55, 1318-1324.

Wyszecki, G., \& Stiles, W. S. Color science. New York: Wiley, 1967.

\section{NOTES}

1. This method is not new. Wyszecki (personal communications) has advocated this method "as the only trustworthy one to be used ...." Indeed, he used it on their seven-field colorimeter (Wyszecki, 1965).

2. Judd's modification of the CIE color-matching function is not a CIE officially sanctioned function. But visual scientists may prefer it because it more adequately represents the average human observer's response below $450 \mathrm{~nm}$. This color-matching function can be found in Wyszecki and Stiles (1967).

3. Spectral radiant power was measured with an E.G.\&G. Spectroradiom eter.

4. No attempt was made to have this white light match CIE Source A or any other specific source. Observers were instructed to produce a white which contained no chromatic content.

(Received for publication May 31, 1974; revision received July 13,1974 .) 